\title{
Lymph node dissection in urological cancers: one topic, many controversies
}

\author{
Avinash Nehra $^{1} \cdot$ R. Jeffrey Karnes ${ }^{1} \cdot$ Giorgio Gandaglia $^{2} \cdot$ Alberto Briganti $^{2}$
}

Received: 12 February 2017 / Accepted: 14 February 2017 / Published online: 1 March 2017

(C) Springer-Verlag Berlin Heidelberg 2017

The current special issue of the World Journal of Urology (WJU) is devoted to lymphadenectomy (LND) in urologic oncology. With the exception of testis and penile cancers, the effect of LND in genitourinary cancers is far to have a clear curative role, mainly due to the lack of solid, prospective evidence. However, LND has certainly a prominent role in accurately staging nodal status and in guiding subsequent use of adjuvant therapy, thus potentially improving disease control. Studies involving LND are evolving and have become more focused not only on its diagnostic and prognostic accuracy but also on its therapeutic effect. However, while retrospective studies have shown that a LND may have a potential survival benefit in certain urological cancers, there continues to be controversy. Indeed, since the majority of data are the result of retrospective evidence, the overall clinical impact of LND, optimal extent of dissection, preoperative detection of lymph node metastasis, and treatment complications continue to be investigated. This special issue of the WJU is aimed at reviewing and assessing LND in the staging and management of tumors involving the kidney, prostate, and urothelial cancer of the bladder and upper urinary tract.

Due to conflicting data in published research among patients with renal cell carcinoma, Drs. Capitanio and Leibovich addressed both "The rationale and role of lymph node dissection in renal cell carcinoma [1]." The review analyzes the findings of the prospective, randomized study, EORTC 30,881, which showed no survival benefit among

Alberto Briganti

briganti.alberto@hsr.it

1 Department of Urology, Mayo Clinic, Rochester, MN, USA

2 Division of Experimental Oncology/Unit of Urology, URI, IRCCS Ospedale San Raffaele, Milan, Italy patients treated with nephrectomy alone as compared to patients receiving nephrectomy with LND [2]. A major limitation of this study was that the majority of patients had low-stage (cT1abN0M0) and low-grade tumors and thus would not receive possible benefit in cancer control with any nodal micro-metastatic disease. Challenges in identifying candidates for LND are also due to the limited ability of conventional imaging in detecting metastases to lymph nodes and the varying anatomical drainage patterns of the renal lymphatic system. The authors concluded that patients with larger tumor size, in particular, locally advanced disease and presence of adverse pathological would likely benefit from LND due to each factor leading to increased risk of lymph node metastases [3].

Among patients with prostate cancer (PCa), presence of metastatic disease involving lymph nodes is an important prognostic factor. Nomograms are frequently used preoperatively to predict lymph node invasion (LNI), and prior studies have shown that cancer-specific survival is significantly improved among patients with low volume of nodal invasion [4]. The European Association of Urology (EAU) guidelines recommend the use of extended pelvic LND among patients with a risk of nodal invasion higher than 5\%, although LND may result in higher complication rates. Unfortunately, current imaging modalities are unable to correctly stage lymph node status at the time of diagnosis of PCa, as described by Dr. Incerti and colleagues [5]. The most used and promising tool for nodal assessment is certainly PET/CT using either choline or prostate-specific membrane antigen (PSMA). While both radiotracers have limited sensitivity in detecting microscopic nodal invasion at the time of diagnosis, PSMA has shown promise in staging recurrent nodal disease at lower PSA values as compared to choline. Further studies are required to correlate 
imaging with PSMA and histologic assessment, various dosing of the radiotracers as well as patient outcomes.

Dr. Conti and collaegues described the importance of histo-pathological evaluation of the entire lymph node specimens among patients with $\mathrm{PCa}$ in order to detect the presence of occult metastatic disease and guide further treatment [6]. Lymph node specimens can be examined by various methods, including frozen section which can be most useful in patients with high-risk disease, total inclusion of the tissue, and large-format histology. This would allow for the detection of more lymph nodes than standard sampling as well as for serial sectioning and use of immunohistochemistry. While serial sectioning of lymph nodes has improved detection rates, its impact is still to be properly assessed in clinical practice having also increased cost. In addition, morphological assessment of affected lymph nodes including information on the size of metastases, extra-nodal extension, and lymph node metastasis in periprostatic/periseminal vesicle fat may help in improving predictions of men with $\mathrm{N}+$ disease.

For muscle-invasive bladder cancer, radical cystectomy with LND with or without neoadjuvant therapy is the gold standard therapy. In this context, LND is important for both staging of disease and guiding subsequent adjuvant therapy. As for $\mathrm{PCa}$, preoperative imaging studies have limited accuracy in detecting microscopic nodal metastasis and therefore histological evaluation remains paramount. It has been established that lymph node involvement is associated with increased risk of recurrence and progression of bladder cancer. Several retrospective evidences have also shown improved survival in patients with greater extent of nodal dissection [7]. However, there is lack of consensus as to the optimal anatomical extent of LND and whether superextended LND can improve patient outcomes. Drs. Hugen and Daneshmand address these issues in "Lymph node dissection in bladder cancer: Where do we stand? [7]." With regard to anatomical boundaries for adequate lymph node dissection, numerous studies have demonstrated that in patients with both $\mathrm{N} 0$ and $\mathrm{N}+$ disease, a more thorough lymphadenectomy leads to improved recurrence-free survival and overall survival. Two randomized controlled trials, one sponsored by the Association of Urogenital Oncology and the other SWOG S1011, are ongoing to determine the extent of lymph node dissection which yields maximal benefit. Lymph node yield can vary based upon surgical technique, surgical template, and reporting standards, but analysis of lymph node yield in patients with $\mathrm{N}+$ disease has shown that the removal of greater numbers of lymph nodes intraoperatively was significantly associated with decreased local recurrence and improvement in recurrencefree survival. Lymph node density has also been shown as a factor predictive of recurrence-free survival and overall survival. Although technically challenging, a minimally invasive surgical approach for lymphadenectomy can be certainly considered among experienced surgeons in order to yield similar results to an open lymphadenectomy.

Upper tract urothelial carcinoma (UTUC) accounts for $5-10 \%$ of all urothelial cancers, and nodal metastasis is again a poor prognostic indicator with significant impact on survival. The treatment for majority of patients is radical nephroureterectomy, and Dr. Seisen and colleagues have analyzed the controversy regarding the role of LND in this setting [8]. Mapping studies have demonstrated the varying patterns of lymph node involvement for tumors affecting the respective renal pelves and differing ureteral segments. A review of the literature not surprisingly demonstrated that patients with $\mathrm{pN}+$ disease had significantly worse cancer-specific survival when compared to patients with $\mathrm{pNO}$ and $\mathrm{pN} x$ disease and also demonstrated that LND, particularly among patients with muscle-invasive disease, provides an accurate staging method. Extra-nodal extension of disease is also an independent predictor of both disease recurrence and cancer-specific mortality and can be utilized to stratify patients for additional treatments. With regard to the therapeutic benefit of LND, retrospective evidence has shown that performing LND in patients with muscle-invasive UTUC was associated with improved cancer-specific survival, especially among patients with locally advanced disease and muscle-invasive UTUC. Furthermore, removal of a greater number of lymph nodes provided value in both detecting nodal involvement and improved cancer-specific survival, but standardized templates for LND have not been developed. Moreover, level 1 evidence is still needed also in this area.

In conclusion, each of the studies described have shown benefits of lymph node dissection in staging of urologic cancer and in potentially improving cancer outcomes. However, questions remain regarding preoperative detection of lymph node metastasis as well as determining the most optimal candidates and templates. Further studies will provide more information to guide decisions and optimize the benefits of lymph node dissection and this special issue of the WJU has taken a step in this direction.

\section{References}

1. Capitanio U, Leibovich BC (2016) The rationale and role of lymph node dissection in renal cell carcinoma. World J Urol. doi:10.1007/s00345-016-1886-3

2. Blom JH, van Poppel H, Marechal JM, Jacqmin D, Schroder FH, de Prijck L et al (2009) Radical nephrectomy with and without lymph-node dissection: final results of European Organization for Research and Treatment of Cancer (EORTC) randomized phase 3 trial 30881. Eur Urol 55:28-34

3. Blute ML, Leibovich BC, Cheville JC, Lohse CM, Zincke H (2004) A protocol for performing extended lymph node dissection using primary tumor pathological features for patients 
treated with radical nephrectomy for clear cell renal cell carcinoma. J Urol 172:465-469

4. Briganti A, Karnes JR, Da Pozzo LF, Cozzarini C, Gallina A, Suardi N et al (2009) Two positive nodes represent a significant cut-off value for cancer specific survival in patients with node positive prostate cancer. A new proposal based on a two-institution experience on 703 consecutive $\mathrm{N}+$ patients treated with radical prostatectomy, extended pelvic lymph node dissection and adjuvant therapy. Eur Urol 55:261-270

5. Incerti E, Mapelli P, Gianolli L, Picchio M (2016) PET imaging for lymph node dissection in prostate cancer. World J Urol. doi:10.1007/s00345-016-1954-8
6. Conti A, Santoni M, Burattini L, Scarpelli M, Mazzucchelli R, Galosi AB et al (2015) Update on histopathological evaluation of lymphadenectomy specimens from prostate cancer patients. World J Urol. doi:10.1007/s00345-015-1752-8

7. Hugen CM, Daneshmand S (2015) Lymph node dissection in bladder cancer: where do we stand?. World J Urol. doi:10.1007/ s00345-015-1751-9

8. Seisen T, Shariat SF, Cussenot O, Peyronnet B, Renard-Penna R, Colin P, Roupret M (2016) Contemporary role of lymph node dissection at the time of radical nephroureterectomy for upper tract urothelial carcinoma. World J Urol. doi:10.1007/ s00345-016-1764-z 